\title{
Energy measurement resources in spins-first and position-first quantum mechanics
}

\author{
Gina Passante \\ Physics Department, California State University Fullerton, 800 N State College Blvd., Fullerton, CA 92831
}

\begin{abstract}
Research into student understanding of quantum mechanics has primarily focused on the identification of student difficulties a nd the d evelopment of $\mathrm{c}$ urriculum that $\mathrm{c}$ an h elp i mprove $\mathrm{s}$ tudent 1 earning. M ost of this work has been performed in courses using a position-first i nstructional $\mathrm{p}$ aradigm. R ecently, there $\mathrm{h}$ as been increase in the number of instructors choosing to present material in an alternate order: spins-first. In this work, we makes steps towards answering the question of whether or not the instructional paradigm has an impact on student thinking about energy measurement. We use a resources lens to analyze the different tools and methods students are using to solve questions.
\end{abstract}

\section{INTRODUCTION}

Energy is one of the most important observables in quantum mechanics (QM). In addition to characterizing quantum systems (such as the hydrogen atom), energy eigenstates and eigenvalues determine the time evolution of the wave function. The teaching of energy in QM can differ greatly, depending on the instructional paradigm. We are interested in what impact the instructional paradigm has on student thinking about energy measurements. The paradigms we investigate are the two most commonly used in undergraduate QM: position-first and spins-first.

In position-first instruction, students are first introduced to the Schrödinger equation through position space wave functions. The second paradigm uses a spins-first approach where students first learn the postulates of QM and the Schrödinger equation in the context of spin-1/2 particles. Each paradigm relies on very different mathematical tools (differential equations for position-first and matrix equations for spins-first) to teach the fundamental concepts. These differences introduce additional degrees of freedom that researchers must take into account when investigating student understanding and developing research-based curriculum.

This paper focuses on the differences in how energy and the Hamiltonian are first taught in both paradigms and how this affects how students think about energy measurements. Most prior research on student understanding of energy measurements has been done in the position-first paradigm and has focused on common students difficulties (for example, [1] and [2]). In this work we look for similarities and differences in student thinking about energy measurements in both paradigms using a resources lens [3].

\section{INTRODUCTION TO ENERGY INSTRUCTION}

In both instructional paradigms, energy is first taught in the context of the Schrödinger equation as the eigenvalue of the Hamiltonian operator. The main difference comes in the form that the Hamiltonian takes.

Energy in Position-first Instruction: Using Griffiths' Introduction to Quantum Mechanics [4] as a guide for the order of instruction in the position-first paradigm, energy and the Schrödinger equation are introduced almost immediately. The only material that precedes it is an introductory chapter on the wave function, which includes position and momentum operators, probability density, and uncertainty. The time-dependent Schrödinger equation is then introduced and separation of variables results in the time-independent Schrödinger equation, commonly referred to as the energy eigenequation. In this context, the connection between the Hamiltonian operator and the energy values is motivated by recognizing that it can be is $-i \hbar \frac{\partial}{\partial x}$. This is the first eigenvalue equation in Griffiths' textbook, and while students have already been introduced to the position and momentum operators, there has not yet been a description of their eigenvalues or eigenfunctions. The textbook then continues to solve for the energy eigenvalues and eigenfunctions for several different potentials, including the infinite square well, finite square well, and harmonic oscillator.

Energy in Spins-first Instruction: In a spins-first course, several weeks are spent learning the postulates of QM in the context of the spin operators. By the time the Schrödinger equation is introduced, eigenvalue equations are familiar. The main difference is that in the spin-1/2 case the Hamiltonian does not have the familiar form of kinetic plus potential, but is the potential energy of a magnetic dipole in an external (uniform) magnetic field: $-\vec{\mu} \cdot \vec{B}$. While this can be motivated as the energy of the system, it is in a much less familiar form.

When solving the Schrödinger equation for spin-1/2 particles, textbooks often use magnetic fields that are oriented in the $x, y$, or $z$-direction. Since the magnetic moment vector is proportional to the vector of spin-matrices, the eigenstates of energy are the same as the eigenstates of spin in the direction of the magnetic field. For example, in the case of an electron in a uniform magnetic field oriented in the $z$-direction, $\vec{B}=B_{0} \hat{z}$, the Hamiltonian for the system is $\omega S_{z}$, where $\omega=\frac{e B_{0}}{m_{e}}$. Because the Hamiltonian is proportional to $S_{z}$, its energy eigenstates are $|+\rangle_{z}$ and $|-\rangle_{z}$. In matrix notation:

$$
H=\frac{\omega \hbar}{2}\left(\begin{array}{cc}
1 & 0 \\
0 & -1
\end{array}\right),|+\rangle_{z}=\left(\begin{array}{l}
1 \\
0
\end{array}\right),|-\rangle_{z}=\left(\begin{array}{l}
0 \\
1
\end{array}\right) \text {. }
$$

At the beginning of any question involving a spin in a magnetic field, it is routine to determine the Hamiltonian, solve for the energy eigenvalues and eigenstates, and then use that information to solve the appropriate question. This is in 
contrast to common position-space energy questions, which generally rely on the recollection of a previously calculated eigenvalue and eigenfunciton.

\section{RESEARCH METHODS}

In this study we will analyze data from two different questions, administered to different populations in courses using different instructional paradigms. Both questions were given in the first half of a junior-level QM course for physics majors, and both ask for the possible outcomes of an energy measurement.

We are using the theoretical lens of resources [3] to analyze student responses to these two questions. We assume that student thinking is composed of small pieces (that we call resource elements) and the connections between them. Each resource element represents a small conceptual or mathematical piece of information that can be assembled in various ways. Student explanations provide us with information about some of the resources they used or activated when answering the question. The context of the question will impact the resources used, and therefore we expect that differences in the questions will lead to variations in the visible resources. By coding student response to questions in both the spin and position contexts, we will be able to draw inferences about student thinking.

\section{A. Question 1: Spins}

The context of question 1 is a spin-1/2 particle in a magnetic field. This is the first context in which energy measurements can be discussed in a spins-first course. This question was asked of 21 students on the midterm exam of a spins-first course using McIntyre's textbook [5]. At this point in the course, students were familiar with both Dirac notation and the matrix representation for spin states and operators. The question (Fig. 1) provides the quantum state for an electron in matrix notation and asks a total of three questions. The first two ask for the outcomes of a measurement of spin in the $y$-direction at $t=0$ and $t>0$. Part c) asks about the possible outcomes of an energy measurement and their probabilities. It is this last part that we are interested in for this study.

Before determining the energy, students must recognize that the Hamiltonian is the operator that corresponds to the energy observable. The Hamiltonian for an electron in a uniform magnetic field in the $z$-direction can be written as $\omega S_{z}$ (where $\omega=\frac{e B_{0}}{m_{e}}$ ). Then the Hamiltonian and energy eigenvectors are the same as those given in Eqn. (1). The possible outcomes of an energy measurement are the eigenvalues of the Hamiltonian, namely $\pm \omega \hbar / 2$. The probability of each is given by $|z\langle \pm \mid \psi\rangle|^{2}=0.5$.
Question 1:

An electron is initially in the state $\frac{1}{\sqrt{2}}\left(\begin{array}{l}i \\ 1\end{array}\right)$. At $t=0$, a constant magnetic field $B_{0}$ is turned on in the $z$-direction. a) A measurement of the $y$-component of the spin is made at $t=0$. What is the probability of measuring spin up in this direction? Explain your reasoning.

b) Suppose that the measurement above was not made. Instead, a measurement of the $y$-component of the spin is made at $t>0$. Does the probability of measuring spin up in this direction depend on time? Explain your reasoning.

c) Suppose the measurements above were not made. What are the possible outcomes of a measurement of the energy and what are the probabilities of each? Explain or show how you determined your answers.

FIG. 1. Energy measurement question in the context of a spin-1/2 particle in a magnetic field. This question was given in a course that uses the spins-first instructional paradigm. Responses from $N=21$ students over one course have been analyzed.

\section{Question 2:}

A particle in an infinite square well is prepared so that its wave function at time $\mathrm{t}=0$ is: $\Psi(x, 0)=0.6 \Psi_{1}(x, 0)+0.8 i \Psi_{2}(x, 0)$ where $\Psi_{1}(x, t)$ and $\Psi_{2}(x, t)$ are the energy eigenstates corresponding to the ground state, with energy $E_{1}=\varepsilon$ and the first excited state, with energy $E_{2}=4 \varepsilon$

Suppose you measured the energy of this particle at time $t_{1}>0$. What value or values would a measurement of the energy yield? Explain your reasoning.

FIG. 2. Energy measurement question asked in a position-first instructional paradigm. Responses from 52 students over two courses have been analyzed.

\section{B. Question 2: Position}

Question 2 (Fig. 2) was given on an ungraded quiz in a QM course using Griffiths' textbook [4]. This question presents students with a wave function written as a sum of two energy eigenstates of the infinite square well. The coefficients in front of each eigenstate are different and one is imaginary. Students are told that the energy eigenstates have energy values of $E_{1}=\varepsilon$ and $E_{2}=4 \varepsilon$.

In this question it is not necessary for students to reason about the Hamiltonian, since the eigenvalues are already given. However, it is important to identify that both $E_{1}$ and $E_{2}$ are both possible results, since each has a non-zero probability of being measured. The probability is given by the square of the magnitude of the coefficient in front of each term. Because the probability is the square of the magnitude, the fact that one coefficient is imaginary does not factor into the solution. 


\section{RESOURCES IDENTIFIED IN STUDENT RESPONSES}

We analyzed student responses to the two questions described in the previous section and coded for the resources that are visible based on students' written work. Both mathematical and conceptual resources were identified in an iterative coding process by the author. For ease of comparison we report the coded rate as the number of times that code appears over the total number of responses analyzed. One limitation of this analysis is that we only have access to the information that students chose to include in their answer. We are therefore blind to other resources that students may be drawing on to answer the question and our results cannot represent a complete picture of all resources students may have activated.

\section{A. Responses: Spins}

On question 1 , only 2 out of 21 students (10\%) answered correctly. Student responses suggest that most students were not confident in how to answer this question, as many of them wrote down several different responses, often including the probability and outcomes of spin measurements (even though this was not asked for in the question). However, in the answers we can see many productive resources that students are using. The resource elements as well as the rate that each element was coded are given in Table I. (Note that a single response can contain more than one resource element.)

TABLE I. Resource elements visible in student responses to question 1 and the percent of students coded to use each. $(N=21)$

\begin{tabular}{lc}
\hline \hline Resource Element & Coded Rate \\
\hline Energy is an eigenvalue & 0.43 \\
\hline Probability is calculated as $|\langle\mid\rangle|^{2}$ & 0.38 \\
\hline Operator associated with energy is the Hamiltonian & 0.38 \\
\hline Hamiltonian for a spin- $1 / 2$ particle in a magnetic & \\
field is $H=-\mu \cdot B$ and/or $H=\omega S_{n}$ & 0.29 \\
\hline Eigenvalues of spin in any direction are $\pm \hbar / 2$ & 0.19 \\
\hline Eigenvalues can be found by solving $|H-\lambda \mathbb{1}|=0$ & 0.14 \\
\hline The probability is the coefficient squared & 0.05 \\
\hline \hline
\end{tabular}

For an example of how these resource elements are coded in student responses, consider the example shown in Fig. 3. This student correctly identified the energy eigenvalues, however their work is not entirely correct. Additionally, the student wrote the correct form of the equation for the probabilities, but did not use the appropriate eigenvectors and did not perform the calculation correctly. Five of the resource elements are coded in this response: energy is an eigenvalue, the operator for energy is the Hamiltonian, $H=\omega S_{z}$, the eigenvalue of spin is $\hbar / 2$, and probability is calculated as $|\langle\mid\rangle|^{2}$. Note that we are not implying that the student understands that energy is an eigenvalue, for example, but rather that the
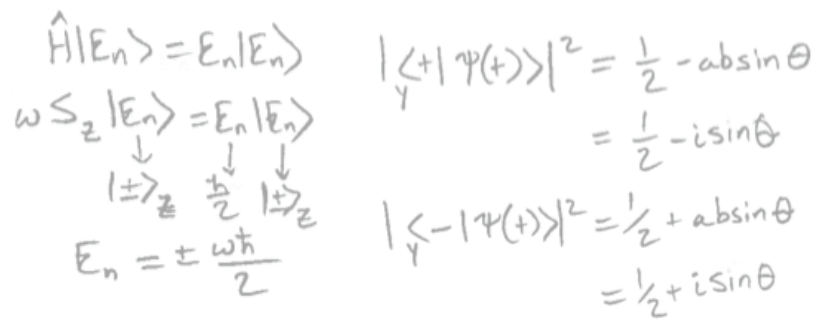

FIG. 3. Student response to question 1c), which asks for the possible outcomes of an energy measurement and their probabilities.

student wrote an eigenequation where $E$ is the eigenvalue and $H$ is the corresponding operator.

\section{B. Responses: Position}

Student performance on question 2 was better (31\% correct), however explanations were more sparse. One potential reason is that students did not need to identify the Hamiltonian or solve for the energy eigenvalues, as they were given them in the question. In addition, this question did not ask students to solve for the probabilities. While some students did reference probabilities as part of their explanation, it was not common. Despite less detailed responses than in question 1 , we were able to code for several resource elements in students' written answers. The elements and their coded rates are given in Table II.

TABLE II. Resource elements visible in student responses to question 2 and the percent of students coded to use each. $(N=52)$

\begin{tabular}{lc}
\hline \hline Resource Element & Coded Rate \\
\hline Multiple values are possible & 0.33 \\
\hline Expectation value & 0.17 \\
\hline The coefficients in the state are meaningful & 0.15 \\
\hline Energy is an eigenvalue & 0.13 \\
\hline Energy cannot be imaginary & 0.10 \\
\hline Operator associated with energy is the Hamiltonian & 0.08 \\
\hline Energy and time have an uncertainty relation & 0.04 \\
\hline The probability is the coefficient squared & 0.04 \\
\hline \hline
\end{tabular}

\section{DISCUSSION}

The goal of this analysis is to see what resources students are activating as they answer questions relating to energy measurements in different contexts. Since energy is taught differently in the two paradigms, we believe students may be building different types of connections between resources in 
each paradigm. This may have implications for how they approach more advanced topics (for example, perturbation theory). In this section we discuss several preliminary findings.

Resources displayed are different: While we expected some differences in the coded resources, we did not expect so few similarities. Only three of the resources overlap. While some of these differences can be explained by the wording of the questions, the results suggest a deeper difference in the data that warrant further investigation.

Resources relating to probability: Although the position question did not ask for probabilities, one of the common coded resources was "the probability is the coefficient squared". While the method for solving for the probability in each question is very different, students in each group gave responses suggesting the activation of a resource in which probability is related to the absolute square of a quantity.

The role of imaginary numbers: The quantum state in both questions contained imaginary coefficients. In the position question, students activated the resource that energy cannot be imaginary. This was given as a reason why $E_{2}$ could not be measured (which is an incorrect answer). No student on the spins question activated this resource, although the same reasoning could be used. For example, it is possible to re-write the matrix notation state given as $\frac{1}{\sqrt{2}}\left(i|+\rangle_{z}+|-\rangle_{z}\right)$, which might lead to a similar response. We plan to provide this representation in a future administration.

Expectation value: Prior research on student difficulties in position-first instructional contexts have identified that students believe the expectation value of a system can be measured [1]. The responses to our position question confirm that some students answered in this way. However, we notice that no student in response to the spins question activated a resource related to the expectation value. It is unclear if this is due to the matrix representation used to describe the state of the particle, or is a true consequence of the instructional paradigm. Future administrations of the question will further test this finding.

Mathematical vs. conceptual resources: The resources identified in this study were more mathematical for the spins question and more conceptual for the position question. This may be due to the different methods required to solve each question, however it is a little surprising since spins-first QM is often taught with a greater focus on the postulates of QM and their conceptual consequences. Additionally, there is preliminary evidence from a different study that students in spins-first courses outperform their peers in position-first courses on a conceptual survey [6]. A similar analysis with different questions that are designed to be isomorphic with respect to calculation type and difficulty will allow us to better investigate if there is a difference in the number of mathematical or conceptual resources used by students.

Role of the Hamiltonian: Students were coded to use the resource that relates the energy to the Hamiltonian operator $38 \%$ of the time in response to the spins question and $8 \%$ of the time on the position question. For position representation questions (such as those in the context of the square well), the energy eigenfunctions (and values) are only calculated once, and the results are simply recalled in future. However, for spin-1/2 particles, the energy eigenfunctions depend on the direction of the magnetic field, and must be determined for each new question. Therefore, students in a spins-first instructional paradigm may form a different, and perhaps a more pronounced, connection between the Hamiltonian and the energy.

\section{CONCLUSIONS}

In this preliminary study we set out to investigate how different instructional paradigms may affect student thinking about energy measurements. The resources lens has illuminated some components in student thinking in each context. We identified more mathematical resources to the spins question that focused on the Hamiltonian and the probabilities, while the position question elicited more conceptual resources. Another interesting finding is that no student in response to the spins question activated the resource that imaginary numbers cannot be measured, even though there was an imaginary coefficient in the question. We are unable to conclude whether these differences are due to the instructional and/or question context, or the question wording. The next steps of this study will include the design of questions for both contexts that represent the quantum states in the same notation and that require similar calculations. These questions will be administered to students in each paradigm, which will allow us to better isolate what differences are the result of the instructional paradigm.

\section{ACKNOWLEDGMENTS}

The author would like to thank Michael Loverude, Steven Pollock, and Homeyra Sadaghiani for invaluable conversations throughout this project.
[1] G. Zhu and C. Singh, Phys. Rev. ST Phys. Educ. Res. 8, 10117 (2012).

[2] G. Passante, P. J. Emigh, and P. S. Shaffer, Phys. Rev. ST Phys. Educ. Res. 11, 20110 (2015).

[3] D. Hammer, American Journal of Physics 68, S52 (2000).

[4] D. J. Griffiths, Introduction to Quantum Mechanics (Pearson Prentice Hall, Upper Saddle River, NJ, 2005).
[5] D. H. McIntyre, Quantum Mechanics (Pearson Education, Inc., San Francisco, CA, 2012).

[6] H. R. Sadaghiani and J. Munteanu, 2015 PERC Proceedings pp. 287-290 (2015). 